\title{
A DUAL-SNAKE MODEL OF HIGH PENETRABILITY FOR ULTRASOUND IMAGE BOUNDARY EXTRACTION
}

\author{
Chung-Ming Chen, ${ }^{*}$ Henry Horng-Shing Lu ${ }^{\dagger}$ and An-Ting HsiaO* \\ *Institute of Biomedical Engineering, National Taiwan University, Taipei, Taiwan; and ${ }^{\dagger}$ Institute of Statistics, \\ National Chiao-Tung University, Hsin-Chu, Taiwan
}

(Received 20 April 2001; in final form 2 October 2001)

\begin{abstract}
Most deformable models require the initial contour to be placed close to the boundary of the object of interest for boundary extraction of ultrasound (US) images, which is impractical in many clinical applications. To allow a distant initial contour, a new dual-snake model promising high penetrability through the interference of the noises is proposed in this paper. The proposed dual-snake model features a new far-reaching external force, called the discrete gradient flow, a connected component-weighted image force, and an effective stability evaluation of two underlying snakes. The experimental results show that, with a distant initial contour, the mean distance from the derived boundary to the desired boundary is less than 1.4 pixels, and most snake elements are within 2.7 pixels of the desired boundaries for the synthetic images with $C N R \geq 1$. For the clinical US images, the mean distance is less than 1.9 pixels, and most snake elements are within 3 pixels of the desired boundaries. (E-mail: chung@lotus.mc.ntu.edu.tw) (c) 2002 World Federation for Ultrasound in Medicine \& Biology.
\end{abstract}

Key Words: Boundary extraction, Ultrasound images, Deformable model, Dual-snake model, Discrete gradient glow, Connected components, Stability.

\section{INTRODUCTION}

Boundary information of the object of interest in an ultrasound (US) image is essential for many clinical applications. It may serve as an important indicator for differentiating a benign lesion from a malignant one. For example, the shape of the lesion contour has been considered as one of the most valuable indices for predicting malignancy of a breast lesion (Skaane and Engedal 1998) and a prostate tumor (Yoshio 1988). It may also be the basis for such quantitative and qualitative analyses as area and volume measurements, tissue characterization, motion analysis, 3-D assessment, and so on, for the objects of interest in the US images. Some examples are volume measurement of the left ventricle (Iwase 1994), characterization of ovarian follicles (Sarty et al. 1998), contour tracking of cardiac structures (Chalana et al. 1996; Mikic et al. 1998) and 3-D evaluation of the degree of vessel obstruction (Haas et al. 2000).

Boundary extraction from US image, however, is a much harder problem than from other image modalities,

Address correspondence to: Chung-Ming Chen, Institute of Biomedical Engineering, College of Medicine, National Taiwan University, \#1, Sec. 1, Jen-Ai Road, Taipei, Taiwan. E-mail: chung@lotus. mc.ntu.edu.tw due to the speckle, the tissue-related textures, and the artefacts resulting from the ultrasonic imaging process. Although these three types of signals may clinically play an important role in making diagnosis, the false edges and the deterioration of edge definition caused by them have seriously degraded the performance of most classical boundary-detection algorithms. To overcome the potential problems induced by these three types of signals in boundary detection, numerous approaches have been proposed to extract the boundary of the object-of-interest in US image. Most of these approaches may be categorized into thresholding (Zimmer et al. 1996), clustering (Boukerroui et al. 1998), mathematical morphology (Thomas et al. 1991; Hamdan et al. 1996), wavelet analysis (Fan et al. 1996), artificial neural network (Kotropoulos 1994), genetic algorithm (Heckman 1996), fuzzy logic (Solaiman et al. 1996), deformable model (Haas 2000; Chen and Lu 2001), etc. The clinical applications involved range from 2-D object analysis (Thomas et al. 1991; Sarty et al. 1998) to image-guided brain surgery (Chen and Lu 2001). Despite the reported excellent performance, boundary extraction from US image generally remains a difficult task because of the complex nature of the US images and the limited generalization capability of most previous algorithms. For example, the 
clustering- or thresholding-based methods may fail for an object-of-interest with an inhomogeneous interior.

As a general boundary-detection technique, recently, the deformable model has been gaining increasing interest for US image analysis due to its attractive properties. First, the deformable model promises a continuous derived contour, closed or open, which makes it a preferred choice to many other edge-based approaches requiring edge-linking processes. Second, the inherent cost-minimization mechanism has allowed the deformable model to incorporate various features, including edge-based and region-based features, to determine the object boundaries. Third, the deformation process is naturally an ideal candidate for contour tracking, which may be for the same object deforming in a time sequence or for a continuous set of 2-D slices belonging to the same 3-D object.

The deformable model, also known as the snake model, the active contour model, the deformable contour, and so on, is a curve (or a surface) that deforms, aiming to minimize a specified energy function subject to some constraints. The energy function is usually composed of the internal energy, characterizing the geometric features of the curve, and the external energy, accounting for the image features. Through energy minimization, the curve deformation is basically guided by the internal and the external forces generated from the internal and the external energies, respectively. The internal force regularizes the length and the smoothness of the curve, and the external force attracts the curve toward the desired boundary. The energy may be computed for all snake elements or only for some selected elements, called "snaxels." Two types of deformable models have been developed previously. One is the parametric deformable model, which formulates the curve explicitly in the parametric form. The other is the geometric deformable model, which implicitly characterizes the curve by a level set function. Generally speaking, the geometric deformable model has the advantage over its counterpart in handling the topological change more naturally, whereas the parametric deformable model tends to be more flexible in imposing varieties of guiding forces for deformation. It has been shown that the implicit model does not perform well in case of poor contrast boundaries or gaps in boundaries (Niessen et al. 1998).

Taking advantage of the attractive properties of the deformable model, many parametric deformable models have been proposed in the past decade to identify the desired object boundaries from various types of US images. Some typical works are those of Pathak et al. (1997), Lefebvre et al. (1998), Sarty et al. (1998), Shekhar et al. (1999), Haas et al. (2000), and so on. Even though these deformable models have achieved reasonable performances for the underlying clinical applica- tions, they suffer from at least one common problem intrinsic to most deformable models, that is, the initial contour generally needs to be placed very close to the desired boundary. This constraint has severely hindered the deformable model from daily clinical use because of the burdensome effort required by the medical doctors to make such initial contours during the clinical work. For most deformable models, this deficiency mainly arises from their inability to escape from the local minimums caused by the speckle, the tissue-related textures or the artefacts while the deforming curve moves toward the desired boundary.

To remedy this deficiency, (i.e., to allow a distant initial contour) at least three classes of approaches have been proposed in the past. The first class of approaches is based on the idea of extending the attraction range of the external force. Kass et al. (1987) and Terzopoulos et al. (1988) suggested increasing the attraction range by computing the external force at different scales. Cohen (1991) proposed a pressure force to deform the curve until it is stopped by the external force. Cohen and Cohen (1993) defined the external force in terms of the distance between the pixel and the nearest boundary point. $\mathrm{Xu}$ and Prince (1998) devised a new external force, called gradient vector flow (GVF), which promises a large attraction range and a better capability of the curve to deform into boundary concavities. Although these approaches may provide an external force capable of attracting a distant initial contour, all of them are inclined to move the curve elements toward the strong edge points on the way to the desired boundary. If these strong edge points are false edge points, the deforming curve may be trapped at the undesired local minimums.

The second class of deformable models, designed to overcome the local minimums and having a high potential to draw a distant initial contour toward the desired contour, is the dual-snake model (Gunn and Nixon 1997; Kerschner 1998; Giraldi et al. 2000). The general idea of the dual-snake model is to employ two snakes, the inner and the outer snakes, instead of one snake during the deformation process. While the outer snake contracts inward, the inner snake expands outward. The two snakes deform individually under the influence of the conventional internal and external forces, as well as the driving force, which is termed the DS-potential force in this paper, characterizing the interaction between the two snakes. With the function of pulling the two snakes toward each other, the DS-potential force equips the dual-snake model to have an excellent capability to eject the weak local minimums, which may be hard to escape from by the conventional (single) deformable model. Nevertheless, the dual-snake model generally suffers from a dilemma in determining the weight of the DSpotential force. Although an insufficient DS-potential 
force may fail to get the snake away from the local minimum, an overwhelming DS-potential force may make the snake cross over the desired boundary.

As the third class of approaches, we have recently proposed a new deformable model, called the discrete snake model, based on the discrete concept (Chen et al. 2000). The idea of the discrete concept is to move the snaxels discretely. More precisely, only the edge points are under consideration for the snaxel movements. It is discrete in the sense that the candidate snaxel positions distribute discretely, in contrast to continuously as in the conventional deformable model. The advantage of the discrete snake model lies in its greater capability to go across the energy barrier made by the noise, and thereby, has better noise immunity. Moreover, it guarantees that all snaxels will converge to the boundary defined by the edge points. Effective as it is, the discrete snake model has a potential problem of high curvatures caused by the discrete jumps of the snaxels from edge point to edge point, which may become a hindrance for snake deformation.

By unifying the advantages of these three classes of approaches, we propose a new dual-snake model that promises a high penetrability for boundary extraction of US images. Three new schemes, which evolved from the discrete concept, have been developed to ameliorate the shortcomings of these three classes of approaches. The first scheme is to reinforce the conventional image force of the object of interest by weighting the edge strength with the population of the connected component to which each edge point belongs. A connected component is defined as a cluster of edge points in which every two edge points are physically connected through a set of edge points in the same cluster. It is expected that the edge points of the desired boundary are more likely to form larger connected components than those of the noise.

The second scheme is a new external force, called discrete gradient flow (DGF), to overcome the potential high curvature problem of the original discrete snake model (Chen et al. 2000). The DGF smoothly and monotonically connects the significant reinforced edge points along the moving path of each snaxel. It has been devised to provide the proposed dual-snake model with a farreaching attraction force toward the desired boundary. The third scheme is to incorporate the concept of stability in the control of snaxel movement. The basic idea is to determine the energy state of a snake, not only using the internal and external energies, but also taking into account how close the snaxels are to the edge points. The stability of the snake has been defined as a function of the distances from the snaxels to the nearest edge points. This scheme is particularly useful in reducing the possibility of a snaxel to be latched by a strong noise, which exerts a very large image force even after it has been weighted by the size of the connected component. The proposed dual-snake model has been shown to be capable of deforming from a distant initial contour on both of the simulated and the clinical US images, which would be a valuable capability for the clinical use.

\section{MATERIALS AND METHODS}

To allow a distant initial contour, a new dual-snake model is proposed in this paper. The proposed dualsnake model not only unifies the power of a new farreaching attraction force, the discrete concept and the attraction force exerted from two snakes, but also greatly alleviates the potential problems of these three classes of approaches.

As a parametric deformable model, the proposed dual-snake model comprises two snakes (i.e., the outer and the inner snakes) which are denoted as $\Gamma_{0}$ and $\Gamma_{1}$, respectively. The energy of each snake is composed of two internal energies, $E_{\text {cont }}$ and $E_{\text {curvature, }}$ one external energy, $E_{D G F}$, weighted by the stability index $S_{1}$, and one DS-potential energy, $E_{D S \text {-potential }}$. All these energies are defined on the snaxels, (i.e., the snake elements selected from each snake). Both snakes have the same number of snaxels. For convenience, the snaxels of the outer and the inner snakes are called the outer and the inner snaxels, respectively. Denote the $i$ th outer snaxel and the $i$ th inner snaxel as $v_{0}\left(s_{i}\right)$ and $v_{1}\left(s_{i}\right)$, respectively. Each pair of $v_{0}\left(s_{i}\right)$ and $v_{1}\left(s_{i}\right)$ have been designed to move toward each other along the straight line connecting both snaxels until they meet together. Let $\Gamma_{l}\left(s_{i}\right)=\left(x\left(v_{l}\left(s_{i}\right)\right), y\left(v_{l}\left(s_{i}\right)\right)\right.$ stand for the coordinate of $v_{l}\left(s_{i}\right)$, where $l=0$ and 1 . The energy function of each snake is defined as:

$$
\begin{gathered}
E_{\text {snake }}\left(\Gamma_{l}\right)=\sum_{\forall v\left(s_{\mathrm{i}}\right) \in \Gamma_{l}}\left[\alpha_{l} E_{\text {cont }}\left(v_{l}\left(s_{i}\right)\right)+\beta_{l} E_{\text {curvature }}\left(v_{l}\left(s_{i}\right)\right)\right. \\
\left.\quad+\gamma_{l} S_{I}\left(v_{l}\left(s_{i}\right)\right) E_{D G F}\left(v_{l}\left(s_{i}\right)\right)+\delta_{l} E_{D S \text {-potential }}\left(v_{l}\left(s_{i}\right)\right)\right]
\end{gathered}
$$

The constants $\alpha, \beta, \gamma$ and $\delta$ are weighting parameters to define the relative importance among different energy terms.

\section{The internal energies}

$E_{\text {cont }}$ and $E_{\text {curvature }}$ are the internal energies accounting for the first- and the second-order order properties of the snake. For each snaxel $v_{l}\left(s_{i}\right)$, define $\vec{u}_{l}\left(s_{i}\right)=\vec{\Gamma}\left(s_{i}\right)-$ $\vec{\Gamma}\left(s_{i-1}\right)$. Then,

$$
E_{\mathrm{cont}}\left(v_{l}\left(s_{i}\right)\right)=\frac{1}{2}\left(\left|\vec{u}_{l}\left(s_{i}\right)\right|+\left|\vec{u}_{l}\left(s_{i+1}\right)\right|\right)
$$

and 


$$
E_{\text {curvature }}\left(v_{l}\left(s_{i}\right)\right)=-\cos ^{-1}\left(\frac{\vec{u}_{l}\left(s_{i}\right) \cdot \vec{u}_{l}\left(s_{i+1}\right)}{\left|\vec{u}_{l}\left(s_{i}\right)\right| \cdot\left|\vec{u}_{l}\left(s_{i+1}\right)\right|}\right)
$$

where $|\vec{a}|$ denotes the 2-norm of the vector $\vec{a}$. For each snake, the sum of all $E_{\text {cont }} \mathrm{s}$ is approximately equal to the length of the snake. To force the outer and the inner snakes to contract inward and expand outward as the $E_{\text {cont }}$ energy is minimized, the former and the latter have been assigned with positive and negative $\alpha$, respectively. On the other hand, the sum of all $E_{\text {curvature }}$ gives a rough measure of the smoothness of each snake. Minimizing the $E_{\text {curvature }}$ energy tends to give a smooth snake contour.

\section{The external energy}

The external energy is usually defined either by such image features as the intensity gradient and the pixel intensity, which has been commonly referred to as the image energy, or by an energy distribution evolved from the image energy. Conventionally, the external energy plays two important roles in snake deformation. One is to serve as a stopping force when a snaxel comes across the desired boundary. The other is to provide an attraction force to guide the deforming curve toward the desired boundary. A far-reaching external force is one of the major keys to guide a distant curve deforming through the interfering attraction of the local minimums formed by the noise. For the first role, however, the conventional external forces generated based on the intensity gradient or the intensity itself may not be strong enough to stop the snaxels crossing over the desired boundary, due to the low signal-to-noise ratio (SNR) of the object of interest in US image. For the second role, although the conventional far-reaching forces, such as the gradient vector flow (Xu and Prince 1998), are capable of guiding the snaxels toward the distant object of interest, the snaxels are easily attracted by the local maximums of the external forces. To demonstrate the potential problem of the conventional far-reaching forces, Fig. 1a shows a synthetic edge image, Fig. 1b and 1c gives the final contour attained by using the GVF (Xu and Prince 1998), and the corresponding GVF field for the upper-left quarter of the image, respectively. Figure 1 is an enlarged edge image, the original size of which is $64 \times 64$. The outermost curve in Fig. 1a is the initial contour, which is a circle centered at $(32,32)$ with the radius of 30 . The desired object boundary is the central circle centered at $(32,32)$ with the radius of 9 . The 13 sporadic dots are to simulate the false edges due to the noise. The programs used to realize the GVF model were obtained from the Web page of $\mathrm{Xu}$ and Prince (http:// iacl.ece.jhu.edu/projects/gvf/). Figure $1 \mathrm{~b}$ shows that the
GVF is able to attract a distant initial contour toward the desired boundary. However, the deforming curve can be easily influenced by the attraction force exerted from the false edges, as illustrated in Fig. 1c and, thereby, captured by the undesirable local minimums.

The fundamental problem of the conventional farreaching external force is that each snaxel can only see the locally minimal external energies in its vicinity and has no idea about whether there is a better minimum beyond them. To overcome this problem, we propose a new far-reaching external force, called the discrete gradient flow $(D G F)$, which aims to provide a monotonically decreasing external energy for each snaxel to escape from a local minimum and move toward a better minimum along its moving path. The $D G F$ may be derived from any conventional edge strength map (e.g., the output of applying a Gaussian filter to the image, followed by the Sobel operator, which gives the edge strength at each pixel). Because the emphasis of this study is on the performance of deformable models, in this work, the modified trimmed mean (MTM) filter has been chosen to remove the speckle for its simplicity and reasonably good denoising capability. The edge strength map is computed by using the Sobel operator. The MTM filter was proposed by Lee and Kassman (1985) in the attempt to combine the advantages of the mean and median filters. Let $W_{i}$ denote the set of pixels in the window centered at the pixel $i$ of the underlying image for speckle reduction and $w_{i}(j)$ the value of the $j$ th pixel in $W_{i}$. Suppose that the number of elements in each window is $N$. Let $m_{i}$ represent the median value of all pixel values in $W_{i}$. Then, given a threshold $t$, the output of the MTM filter, denoted as $y_{i}$ for the pixel $i$ is defined as:

$$
y_{i}=\sum_{j=1}^{N} a(j) w_{i}(j) / \sum_{j=1}^{N} a(j)
$$

where

$$
a(j)=\left\{\begin{array}{l}
1, \text { if }\left|w_{i}(j)-m_{i}\right|<t \\
0, \text { otherwise }
\end{array}\right.
$$

To construct the $D G F$ map, the peaks of the given edge strength map are first determined, which define the candidate edge points. To eliminate spurious edge points, those peaks with the edge strengths smaller than the mean of the edge strengths within the region of interest (i.e., the area enclosed by the outer snake) are excluded. Let $L_{i}$ and $p_{j}^{i}$ denote the moving path of the snaxel $v_{l}\left(s_{i}\right)$ and the $j$ th point in $L_{i}$, respectively. The moving path $L_{i}$ is defined starting from the initial 


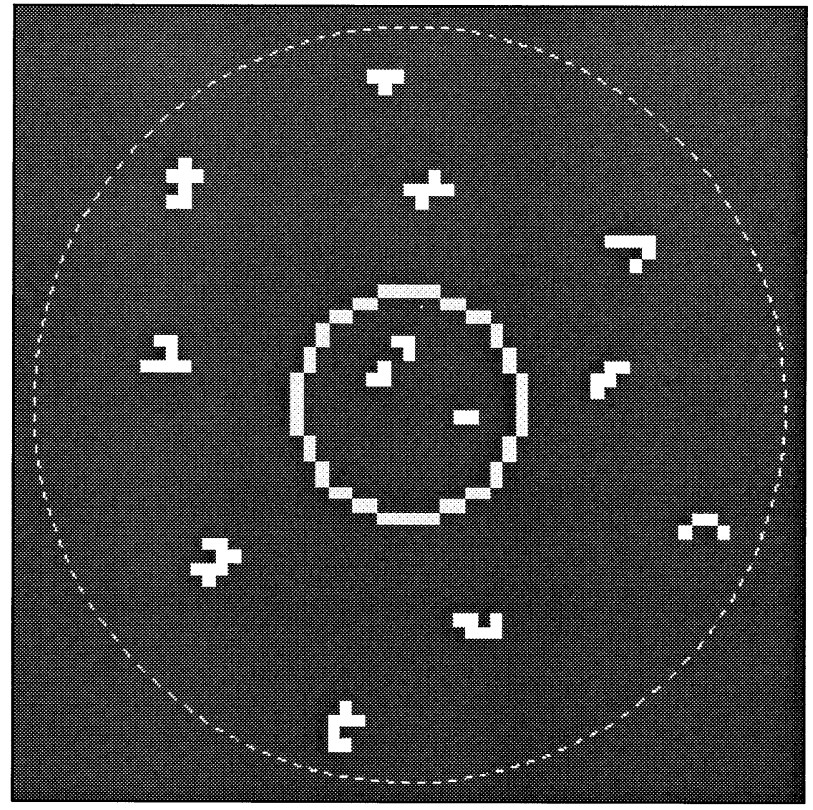

(a)

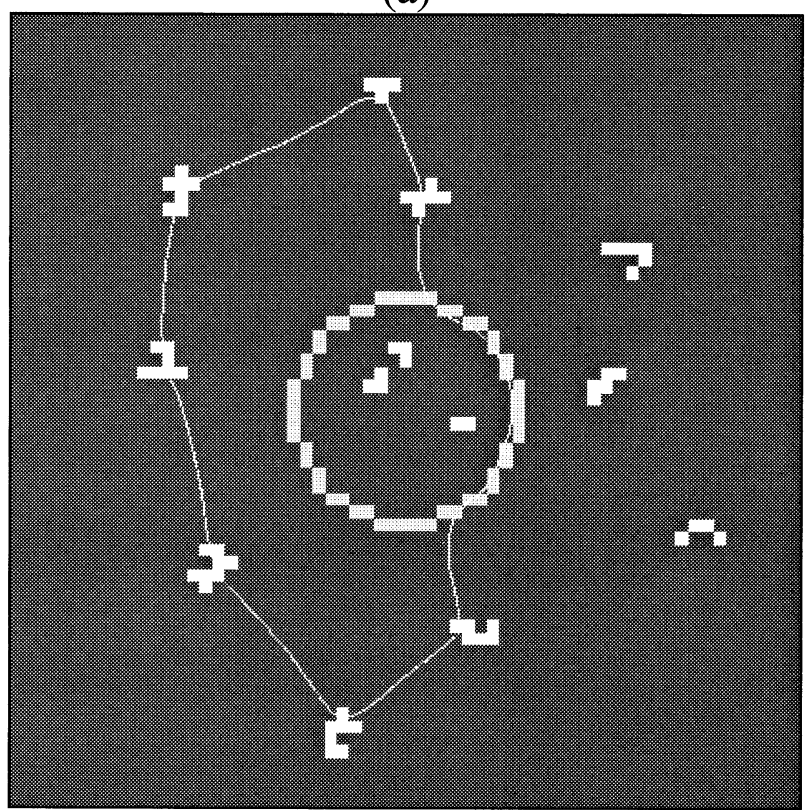

(b)

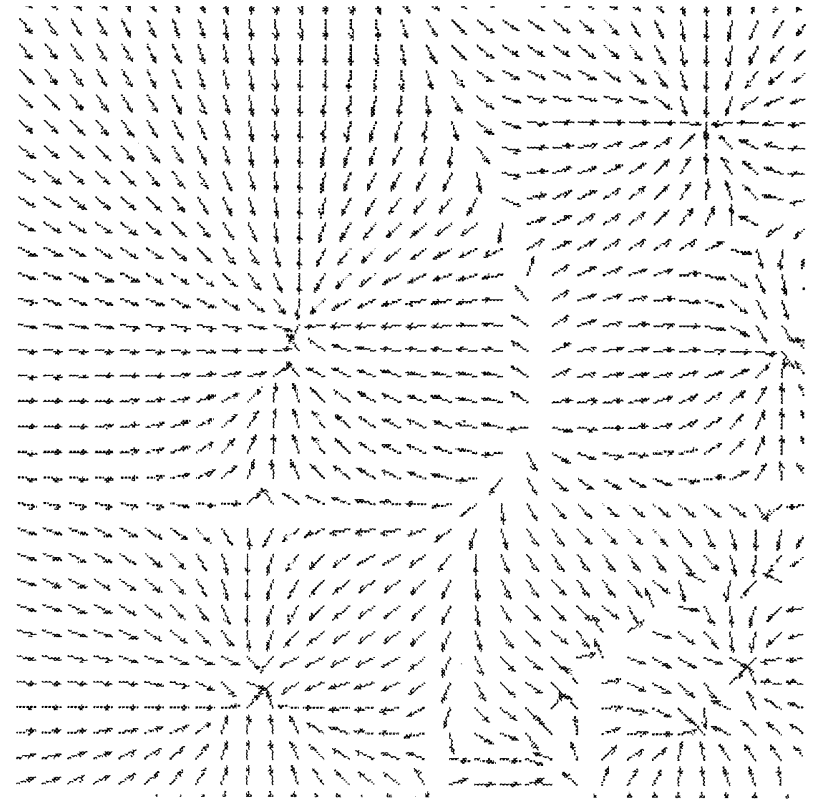

(c)

Fig. 1. (a) A synthetic image in which the central circle is the desired object boundary and the outermost circle is the initial contour of the GVF snake; (b) the deformation result attained by the GVF snake; (c) the GVF field for the upper-left quarter of the image.

contour of the outer snake (i.e., $p_{1}^{i}$, and ending at the initial contour of the inner snake, $p_{m}^{i}$, assuming $L_{i}$, consists of $m_{i}$ points). Suppose $L_{i}$ passes through $n_{i}$ edge points, which correspond to the set of points $\Omega_{i}=\left\{p_{k 1}^{i}, p_{k 2}^{i}, \ldots, p_{k n_{i}}^{i}\right\}$ in $L_{i}$, where $1 \leq k 1 \leq k 2$ $<\cdots<k n_{i} \leq m_{i}$. Let $e\left(p_{j}^{i}\right)$ represent the edge strength of the point $p_{j}^{i}$. Then, for each $p_{j}^{i} \in \Omega_{i} \cup\left\{p_{1}^{i}, p_{m_{i}}^{i}\right\}$, recursively eliminate $p_{j}^{i}$ if $e\left(p_{j}^{i}\right)<e\left(p_{j l}^{i}\right)$ and $e\left(p_{j}^{i}\right)<$ $e\left(p_{j r}^{i}\right)$, where $p_{j l}^{i}, p_{j r}^{i} \in \Omega_{\mathrm{i}} \cup\left\{p_{1}^{i}, p_{m_{i}}^{i}\right\}$, and $p_{j l}^{i}$ and $p_{j r}^{i}$ are the nearest neighbors to the left and to the right of $p_{j}^{i}(i . e ., j l<j<j r)$. Let $\Lambda_{i}=\left\{p_{d 1}^{i}, p_{d 2}^{i}, \ldots, p_{d n_{i}}^{i}\right\}$ denote the set of remaining points in $\Omega_{i} \cup\left\{p_{1}^{i}, p_{m_{i}}^{i}\right\}$ after elimination, where $1 \leq d 1<d 2<\cdots<d n_{i} \leq m_{i}$. The DGF for all points in $L_{i}$ is defined as: 


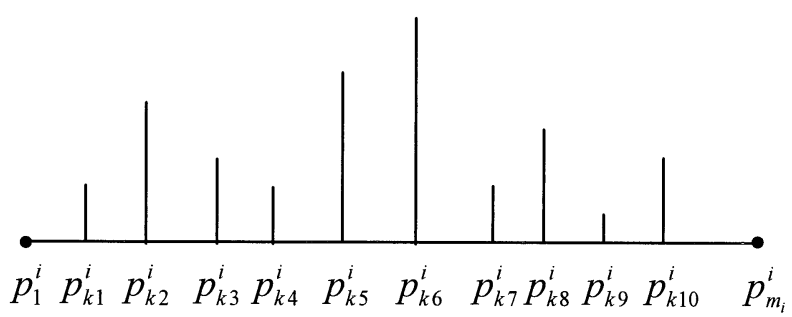

(a)

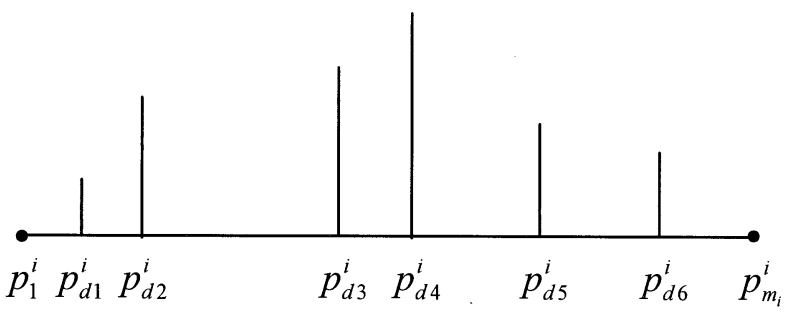

(b)

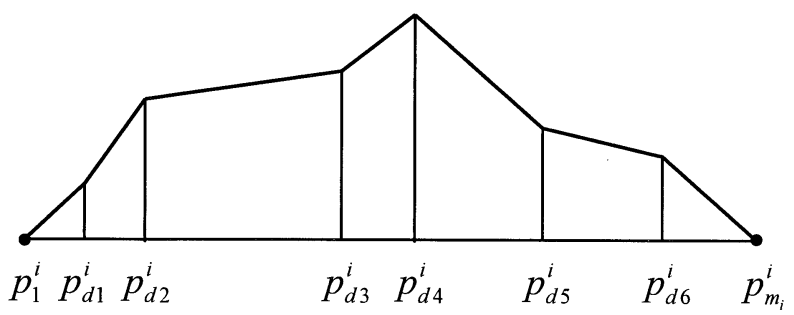

(c)

Fig. 2. (a) An example of candidate edge point set in $L_{i}$ i.e., $\Omega_{i}$ $\cup\left\{p_{1}^{i}, p_{m_{i}}^{i}\right\}$; (b) the edge point set for constructing the $D G F$ on $L_{i}\left(\right.$ i.e., $\left.\Lambda_{i}\right)$; and (c) the $D G F$ on $L_{i}\left(\right.$ i.e., $\left.D G F_{i}\right)$. In these three figures, the height of the vertical line segment at each edge point, $p_{j}^{i}$, represents the edge strength $e\left(p_{j}^{i}\right)$.

$$
D G F_{i}\left(p_{j}^{i}\right)=\frac{d\left(p_{j l}^{i}, p_{j}^{i}\right)}{d\left(p_{j l}^{i}, p_{j r}^{i}\right)} e\left(p_{j r}^{i}\right)+\frac{d\left(p_{j}^{i}, p_{j r}^{i}\right)}{d\left(p_{j l}^{i}, p_{j r}^{i}\right)} e\left(p_{j l}^{i}\right)
$$

where $p_{j l}^{i}, p_{j l}^{i} \in \Lambda_{i}$, and $p_{j l}^{i}$ and $p_{j r}^{i}$ are the nearest neighbors to the left and to the right of $p_{j}^{i}$, and $\mathrm{d}\left(p_{j 1}^{i}, p_{j 2}^{i}\right)$ denotes the Euclidean distance between $p_{j 1}^{i}$ and $p_{j 2}^{i}$ in $L_{i}$. Then, the $E_{D G F}\left(v_{l}\left(s_{i}\right)\right)$ at the point $p_{j}^{i}$ in $L_{i}$ is defined as $D F G_{i}\left(p_{j}^{i}\right)$.

As an example, Fig. 2a-c illustrates $\Omega_{i} \cup\left\{p_{1}^{i}, p_{m_{i}}^{i}\right\}$, $\Lambda_{i}$ and $D G F_{i}$, respectively, with $k n_{i}=10$ and $d n_{i}=6$. The height of the vertical line segment at each edge point, $p_{j}^{i}$ in Fig. $2 \mathrm{a}-\mathrm{c}$ represents the edge strength $e\left(p_{j}^{i}\right)$. To obtain Fig. 2b from Fig. 2a, two iterations of edge point removal are required. In the first iteration, the edge points $p_{k 4}^{i}, p_{k 7}^{i}$, and $p_{k 9}^{i}$ are removed because $e\left(p_{k j}^{i}\right)<$ $e\left(p_{k(j-1)}^{\mathrm{i}}\right)$ and $e\left(p_{k j}^{i}\right)<e\left(p_{k(j+1)}^{\mathrm{i}}\right)$, where $j=4,7$ and 9. In the second iteration, $p_{k 3}^{i}$ is removed because $e\left(p_{k 3}^{i}\right)<$ $e\left(p_{k 2}^{i}\right)$ and $e\left(p_{k 3}^{i}\right)<e\left(p_{k 5}^{i}\right)$, which gives Fig. $2 \mathrm{~b}$.
Conceptually, the $D G F$ has been constructed implicitly based on the discrete concept. That is, only the edge points are considered as the candidate positions for the snaxels to stop at. However, unlike the discrete snake model (Chen et al. 2000), which moves a snaxel from edge point to edge point discretely, the $D G F$ offers a smooth transition between every two significant edge points.

Although the $D G F$ provides an effective far-reaching force to penetrate the interference of the noise and to guide a snaxel toward the desired boundary, the success of the $D G F$ heavily depends on the edge strength of the desired boundary. It is because the $D G F$ intrinsically attracts a snaxel toward a significant edge point. But note that it does not necessarily mean that a snaxel will always converge to the strongest edge point because of the compromise among all energies involved. To augment the edge strength of the object of interest produced by the conventional approaches, we propose a new weighting scheme based on the concept of connected components that may be applied to any conventional edge-strength map. The idea is to cluster the candidate edge points that may be derived from any conventional edge strength map, into connected components. A connected component is defined as a cluster of edge points $C=\left\{p_{c 1}\right.$, $\left.p_{c 2}, \ldots, \mathrm{p}_{\mathrm{cn}_{\mathrm{c}}}\right\}$ in which every two edge points are physically connected through a set of edge points in the same cluster. In other words, one may travel from every edge point $p_{c i} \in C$ to every other edge point $p_{c j} \in C$ through a set of edge points in $C$. Note that the union of two connected components is not a connected component. It means that one cannot travel from any edge point in one of these two connected components to any edge point in the other connected component without going through the non-edge points. Taking Fig. 1a as an example, the central circle of radius 9 , (i.e., the desired boundary of the object of interest) is a connected component and each of the 13 sporadic dots is also a connected component.

With the assumption that an edge point in the desired boundary is expected to be physically connected to more edge points than a false edge point, the strength of each candidate edge point is weighted by the size of the connected component to which the edge point belongs. The size of a connected component is defined as the number of edge points in the connected component. To minimize the noise effect on breaking a connected component into small pieces, in practical implementation, the connected components are determined on the morphologically dilated edge map using a $3 \times 3$ square structuring element. The $3 \times 3$ square structuring element is defined as:

$$
\left[\begin{array}{lll}
1 & 1 & 1 \\
1 & 1 & 1 \\
1 & 1 & 1
\end{array}\right]
$$




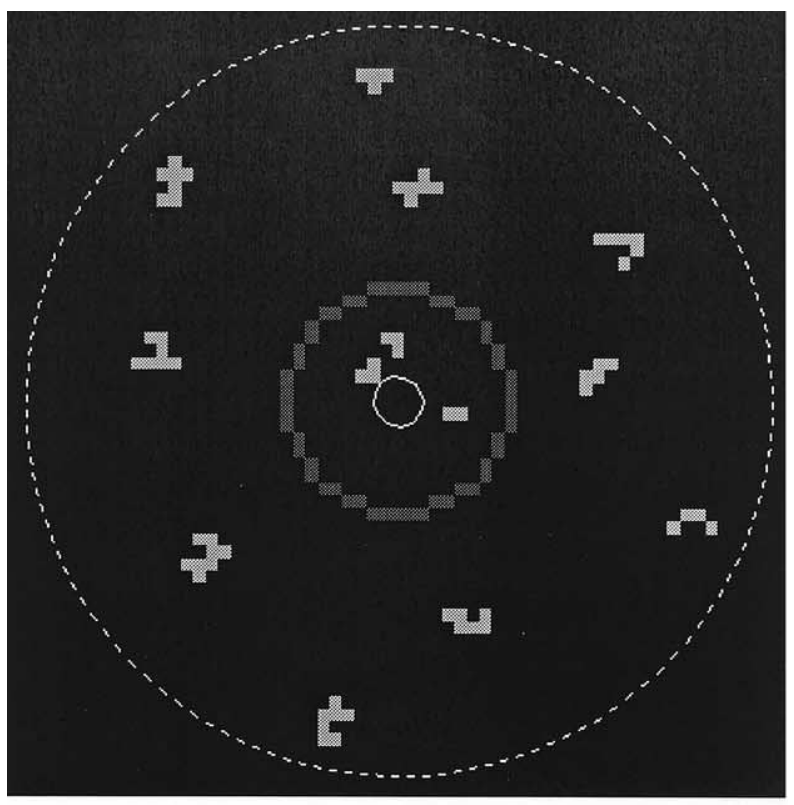

(a)

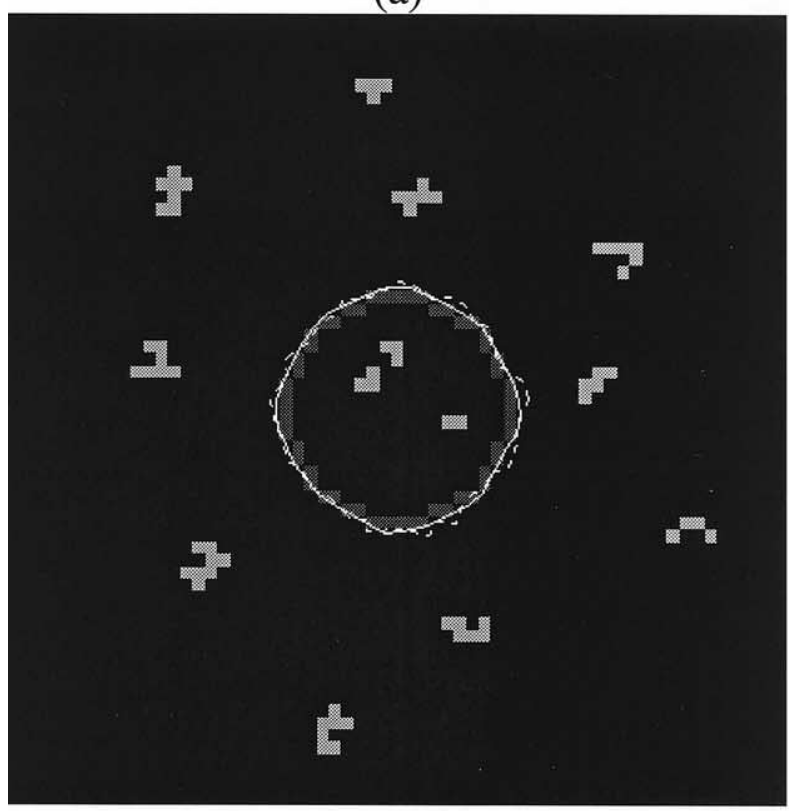

(b)

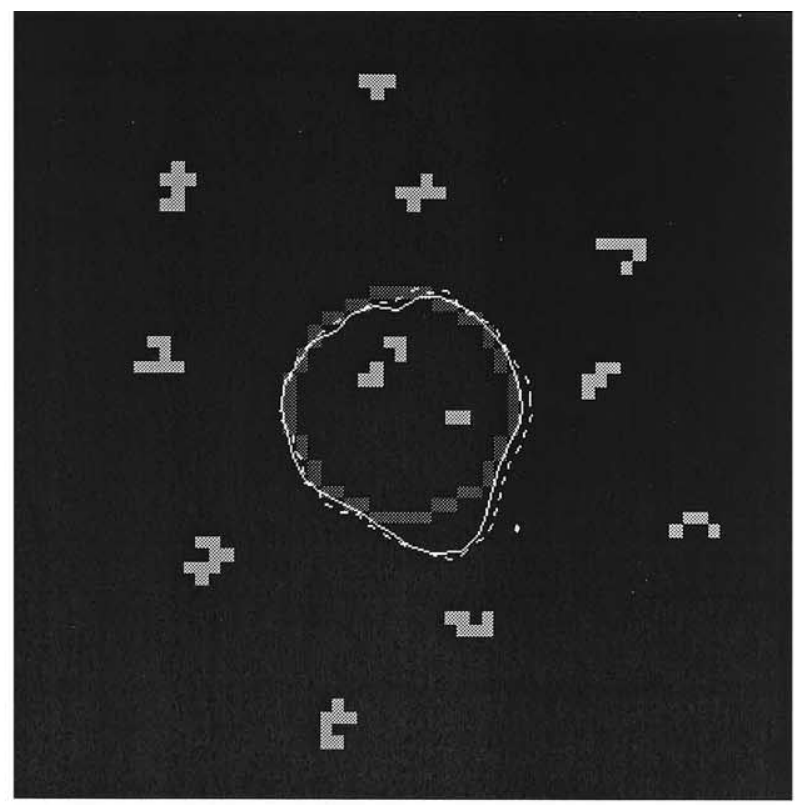

(c)

Fig. 3. (a) A synthetic image in which the central circle is the desired object boundary, the outermost and the innermost circles are the initial outer and inner contours of a dual snake model; (b) both snakes may converge to the desired contour if the weight of the DS-potential force is properly controlled; (c) the snakes may deform to an undesired result if a slightly larger weight than that used in (b) is adopted.

\section{DS-potential energy and snake stability}

The advantage of using dual snakes over using a single snake is that the dual-snake model provides a chance for a snake to escape from a weak local minimum by using the attraction force between two snakes (i.e., the DS-potential force). The DS-potential force usually pushes the snake with the higher energy (i.e., less stable) toward the other one with the lower energy. Nevertheless, control of the DS-potential force is by no means a simple issue. As an example, Fig. $3 b$ and $3 c$ illustrate the deformation results using the dual-snake model modified from Kerschner's approach (Kerschner 1998) for the 
synthetic edge image shown in Fig. 1a. The initial contours of the dual-snake model are provided in Fig. 3a, in which the dashed-line and the solid-line circles are the outer and inner snakes, respectively. Both circles are centered at $(32,32)$. The radii of the dashed-line and the solid-line circles are 30 and 2, respectively. Although the dual snake models for Fig. $3 b$ and $3 c$ are basically the same, except that the weight of the DS-potential force used for Fig. $3 b$ is slightly larger than that for Fig. 3c, the results are quite different. In Fig. 3b, both snakes successfully penetrate the interference of the noise and converge to the vicinity of the desired boundary. But, in Fig. $3 \mathrm{c}$, the inner snake has passed over the desired boundary due to the large contraction force from the outer snake.

To overcome the potential problem of conventional dual-snake model, in this paper, the snake deformation in the proposed dual-snake model has been decomposed into three stages. In the first stage, the two snakes move toward each other independently until both snakes reach the local minimums based on the internal energies, $E_{\text {cont }}$, $E_{\text {curvature }}$, and the external energy, $E_{D G F}$. The goal of the first stage is to guide both snakes as close to the desired boundary as possible, which implies that the external energy, $E_{D G F}$, should serve as the major force to overcome the interference of the false edges for both snakes. Therefore, for all snaxels in this stage, the stability index $S_{\mathrm{I}}$ is set to $1, \delta$ is set to 0 and $\gamma$ should be sufficiently larger than $\alpha$ and $\beta$ so that $E_{D G F}$ may dominate the snake deformation.

After both snakes come across the local minimums, in the second stage, the stability of each pair of the outer and the inner snaxels, $v_{0}\left(s_{i}\right)$ and $v_{1}\left(s_{i}\right)$, is evaluated. The less stable snaxel is encouraged to move forward to the other one by including another energy term, (i.e., the $D S$-potential energy) denoted as $E_{D S \text {-potential }}$ For each snaxel, $v_{l}\left(s_{i}\right), l=0$, or 1 , the $D S$-potential energy is defined as:

$$
E_{D S \text {-potential }}\left(\boldsymbol{v}_{l}\left(s_{i}\right)\right)=\frac{d\left(\boldsymbol{v}_{0}\left(s_{i}\right), \boldsymbol{v}_{l}\left(s_{i}\right)\right)}{\max _{j}\left(d\left(p_{1}^{j}, p_{m_{j}}^{j}\right)\right)} .
$$

The denominator of eqn (8) is a normalization factor to ensure $0 \leq E_{D S \text {-potential }}\left(v_{l}\left(s_{i}\right)\right) \leq 1$ for all snaxels. Because $E_{D S \text {-potential }}\left(v_{l}\left(s_{i}\right)\right)$ is proportional to the Euclidean distance between $v_{0}\left(s_{i}\right)$ and $v_{1}\left(s_{i}\right)$, minimization of the $E_{D S \text {-potential }}\left(v_{l}\left(s_{i}\right)\right)$ gives a great incentive for the less stable snaxel to move toward the other, especially when $v_{0}\left(s_{i}\right)$ and $v_{1}\left(s_{i}\right)$ are far apart from each other.

Determination of the snake stability plays a decisive role for the success of a dual-snake model. Conventionally, the snake stability is evaluated based on the internal and the external energies (Gunn and Nixon 1997). Because the energy is usually computed only for the snax-

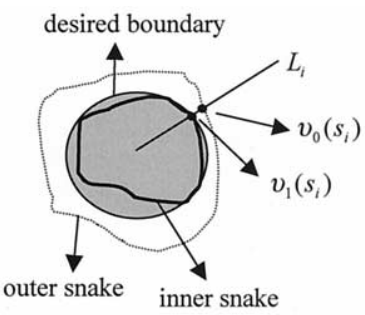

(a)

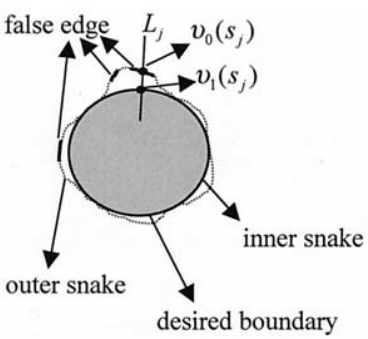

(b)
Fig. 4. (a) On the $i$ th moving path, $v_{1}\left(s_{i}\right)$ has arrived at the desired boundary, which is a circle. But $v_{0}\left(s_{i}\right)$ is still far away from the desired destination. If $v_{0}\left(s_{i}\right)$ happens to be on a strong noise point, $v_{1}\left(s_{i}\right)$ may be determined to be less stable than $v_{0}\left(s_{i}\right)$ and moves away from the desired boundary. (b) On the $j$ th moving path, $v_{0}\left(s_{j}\right)$ and $v_{1}\left(s_{j}\right)$ arrive at a false edge point and a desired edge point, respectively, and both of them are locally stable. If $v_{0}\left(s_{j}\right)$ has a very strong $E_{D G F}$ and only the local stability index and the local energy are taken into account, $v_{1}\left(s_{j}\right)$ may still be attracted toward $v_{0}\left(s_{j}\right)$, though the outer snake is globally less stable.

els, the accuracy of the stability measurement based on the external energy is easily corrupted by the noise. As illustrated in Fig. 4a, the outer dotted and the inner thick curves represent the outer and the inner snakes, respectively. On the $i$ th moving path, $v_{1}\left(s_{i}\right)$ has arrived at the desired boundary, which is a circle. But $v_{0}\left(s_{i}\right)$ is still far away from the desired destination. Ideally, if the energy of $v_{0}\left(s_{i}\right)$ is higher than that of $v_{1}\left(s_{i}\right), v_{0}\left(s_{i}\right)$ will be considered to be less stable and move toward $v_{1}\left(s_{i}\right)$. Nevertheless, if $v_{0}\left(s_{i}\right)$ happens to be on a strong noise point, $v_{1}\left(s_{i}\right)$ may be determined to be less stable than $v_{0}\left(s_{i}\right)$ and moves away from the desired boundary. To overcome this problem, we propose a new stability index based on the discrete concept (i.e., the closer a snake element is to an edge, the more stable the snake element would be). For the $k$ th snake element $v_{1}(k)$ in the snake $\Gamma_{l}, l=0$, or 1, define:

$$
D_{-1}\left(v_{l}(k)\right)=\left\{\begin{array}{cl}
1 & d_{e}\left(v_{l}(k)\right) \leq 2 \\
\frac{1}{d_{e}\left(v_{l}(k)\right)-1} & d_{e}\left(v_{l}(k)\right)>2
\end{array},\right.
$$

where $d_{e}\left(v_{l}(k)\right)$ is the distance from $v_{l}(k)$ to the nearest candidate edge point. Then, the stability index of the snake element $v_{l}(k)$ is defined as:

$$
S_{I}\left(\boldsymbol{v}_{l}(k)\right)=\frac{1}{\left(2 n_{s}+1\right)} \sum_{j=-n_{s}}^{n_{s}} D_{-1}\left(\boldsymbol{v}_{l}(k+j)\right)
$$

where $n_{s}$ defines the radius of the neighborhood. $S_{I}\left(v_{l}(k)\right)$ is basically a measure of how close the neighboring 
snake elements of $v_{l}(k)$ are to the edge points. Note that the stability index is defined for every snake element in a snake, not only for the snaxels. Based on the stability index, for each snaxel $v_{l}\left(s_{i}\right), l=0$, or 1, the stability of $v_{l}\left(s_{i}\right)$, denoted as $S\left(v_{l}\left(s_{i}\right)\right)$, is defined as:

$$
\begin{aligned}
S\left(v_{l}\left(s_{i}\right)\right)= & \bar{S}_{l} S_{I}\left(v_{l}\left(s_{i}\right)\right)\left(\beta E_{\text {curvature }}\left(v_{l}\left(s_{i}\right)\right)\right. \\
& \left.-\gamma w_{D G F}\left(v_{l}\left(s_{i}\right)\right) E_{D G F}\left(v_{l}\left(s_{i}\right)\right) \bar{E}_{D G F-l}\right)
\end{aligned}
$$

where

$$
\begin{gathered}
w_{D G F}\left(v_{l}\left(s_{i}\right)\right)=1-\frac{1}{1+\sqrt{d\left(v_{0}\left(s_{i}\right), v_{1}\left(s_{i}\right)\right)}}, \\
E_{D G F-1}=\frac{1}{N_{L}} \sum_{i=1}^{N_{L}} E_{D G F}\left(v_{l}\left(s_{i}\right)\right),
\end{gathered}
$$

and $\bar{S}_{l}$ the mean of the stability indices of all snake elements in the snake $\Gamma_{l}$, and $N_{L}=$ the number of moving paths.

Unlike the conventional dual-snake models in which the stability of each snaxel is evaluated mostly based on the snake energies, four types of information have been incorporated into $S\left(v_{l}\left(s_{i}\right)\right)$; refer to eqn (11). The first one is the local snake energies, including $E_{\text {curvature }}$ and $E_{\mathrm{DGF}}$. The second one is the local stability index. Incorporation of the stability index is to prevent $v_{l}\left(s_{i}\right)$ from being latched by the strong noise. The rationale of incorporating the stability index is even if a snaxel $v_{l}\left(s_{i}\right)$ is trapped by a false edge point with a high $E_{D G F}$, its neighboring snake elements are very likely not on the candidate edge points. It means that the stability index of $v_{l}\left(s_{i}\right), S_{I}\left(v_{l}(k)\right)$, would be low and, thereby, its stability, $S\left(v_{l}\left(s_{i}\right)\right)$, would be low. The third one is an adaptive weighting factor, $w_{D G F}\left(v_{l}\left(s_{i}\right)\right)$, employed to deemphasize the role of the far-reaching force $E_{D G F}$ as $v_{0}\left(s_{i}\right)$ and $v_{l}\left(s_{i}\right)$ are approaching each other, which would allow the snake to converge to a smoother curve.

The last one is the global property of each snake (i.e., $\bar{S}_{l}$ and $\bar{E}_{D G F-l}$ ) which is very important to remedy the potential problems due to the autonomous deformation of each snaxel. As illustrated in Fig. 4b, consider a scenario that all snake elements of the inner snake represented by the thick circle are right on the desired boundary. Moreover, only some snake elements of the outer snake indicated by the dotted curve are on the candidate edge points. Some of these candidate edge points are the desired edge points and the others are the false edge points, which are marked as the short thick line segments in Fig. $4 \mathrm{~b}$. Suppose $v_{0}\left(s_{i}\right)$ and $v_{1}\left(s_{i}\right)$ on the $j$ th moving path arrive at a false edge point and a desired edge point, respectively. Because the neighboring snake elements of $v_{0}\left(s_{i}\right)$ and $v_{1}\left(s_{i}\right)$ are all on the candidate edge points, the stability indices of $v_{0}\left(s_{j}\right)$ and $v_{1}\left(s_{j}\right)$ are both equal to 1 , (i.e., $v_{0}\left(s_{i}\right)$ and $v_{1}\left(s_{j}\right)$ are both locally stable) If $v_{0}\left(s_{j}\right)$ has a very strong $E_{D G F}$ and only the local stability index and the local energy are taken into account, $v_{1}\left(s_{j}\right)$ may still be attracted toward $v_{0}\left(s_{j}\right)$, though the outer snake is globally less stable. This problem may be partially solved by including the global property, $\bar{S}_{l}$ and $\bar{E}_{D G F-l}$, in the stability evaluation, which would favor the globally more stable snake (i.e., the inner one, in this case)

For each pair of $v_{0}\left(s_{i}\right)$ and $v_{1}\left(s_{i}\right)$, one snaxel, say $v_{0}\left(s_{i}\right)$, is considered to be less stable than the other, say $v_{1}\left(s_{i}\right)$, if $S\left(v_{0}\left(s_{i}\right)\right)<S\left(v_{1}\left(s_{i}\right)\right)$ and:

$$
\frac{S\left(v_{1}\left(s_{i}\right)\right)-S\left(v_{0}\left(s_{i}\right)\right)}{S\left(v_{1}\left(s_{i}\right)\right)+S\left(v_{0}\left(s_{i}\right)\right)}>t_{s}
$$

where $t_{s}$ is a predefined threshold. In this case, $v_{0}\left(s_{i}\right)$ will try to move toward $v_{1}\left(s_{i}\right)$ while $v_{1}\left(s_{i}\right)$ remains at its current position. However, $v_{0}\left(s_{i}\right)$ is allowed to move only if $E_{\text {snake }}\left(\Gamma_{0}\right)$, as defined in eqn (1), decreases. Note that $E_{D G F}$ is weighted by the stability index in this stage, rather than set to 1 as in the first stage. If eqn (14) is not satisfied, both $v_{0}\left(s_{i}\right)$ and $v_{1}\left(s_{i}\right)$ will stay at the current positions until their relative stability changes. The condition imposed by eqn (14) is to avoid the unnecessary snaxel movement due to a small stability difference. In case none of the snaxel pairs satisfies eqn (14), $t_{s}$ may be gradually reduced to relax the condition. Furthermore, if none of the snaxel can move because $E_{\text {snake }}\left(\Gamma_{0}\right)$ does not decrease any more, $\delta$ may be increased little-by-little to augment the attraction force between two snakes.

The third stage is an optional fine-tuning stage that aims to make the derived curve smoother. By using the result of the second stage, one may apply any deformable model to derive a smoother final curve. In this work, we simply turn off the forces exerted from $E_{\text {cont }}$ and $E_{D S \text {-potential, }}$ and set $\beta=-\gamma=1$ and $S_{l}=1$. Then, each snaxel seeks for a lower energy state along its moving path until $95 \%$ of snaxels become stabilized.

\section{The dual-snake algorithm}

As a summary, the proposed dual-snake model has been constructed based on four essential schemes, each of which plays a unique role in assisting snake deformation. First of all, the connected component has been incorporated to provide a better image force. Assuming that the edge points of the desired boundary are more likely to aggregate into larger connected components than the edge points of the noise, it is expected that the 
image force of the desired boundary would be enhanced more substantially than the noise. With the better image force, the DGF and the dual-snake model endow the snake deformation with the higher capability to escape from the undesirable local minimums during the energy minimization process. Although the DGF offers a farreaching force that can penetrate the locally maximal attraction force exerted from the noise, the dual-snake model provides an effective mechanism to eject the undesirable local minimums by cross-referencing the energy states of two snakes. Last, local and global stability have been designed to deemphasize the strong $D G F$ s of the isolated noises and to determine the direction of the attraction force between every pair of the inner and the outer snaxels.

Given an initial contour enclosing the object-ofinterest, the proposed dual-snake model may be summarized as follows:

1. Define the given initial contour as the outer snake $\Gamma_{0}$. Define the area enclosed by $\Gamma_{0}$ as the region-ofinterest (ROI). The initial inner snake, $\Gamma_{1}$, will start as a single point located at the center of mass of the ROI.

2. Construct $N_{L}$ moving paths, each with both ends on $\Gamma_{0}$ and $\Gamma_{1}$.

3. Generate the edge map containing the candidate edge points by any preferred edge detector. Dilate the edge map with a $3 \times 3$ structuring element. Determine the connected component and weight the strength of each edge point by the size of the connected component to which the edge point belongs.

4. Construct the $D G F$ for each moving path.

5. In the first stage, both snakes deform independently until stabilized with $S_{I}=1, \delta=0$, and $\gamma$ sufficiently larger than $\alpha$ and $\beta$ in computing the snake energy $E_{\text {snake }}$ defined in eqn (1). In case any $v_{0}\left(s_{i}\right)$ crosses over $v_{1}\left(s_{i}\right)$, switch $v_{0}\left(s_{i}\right)$ and $v_{1}\left(s_{i}\right)$.

6. In the second stage, compute the stability index using eqn (10) for each snake element in the two snakes. Compute the stability using eqn (11) for each pair of snaxels, $v_{0}\left(s_{i}\right)$ and $v_{1}\left(s_{i}\right)$. If one snaxel is less stable than the other and the condition given in eqn (1) is satisfied, move the less-stable snaxel toward the other. If none of the snaxels can move before both snakes meet together, adjust $t_{s}$ and $\delta$ accordingly.

7. In the third stage, set $\alpha=\delta=0, \beta=-\gamma=1$ and $S_{I}$ $=1$. Then, each snaxel seeks for a lower energy state along its moving path until $95 \%$ of snaxels become stabilized. This is an optional stage.

\section{Images for performance analysis}

Two types of images are to be employed to evaluate the performance of the proposed dual-snake model. One is the synthetic image and the other is the clinical US image. Because the desired boundary of each synthetic

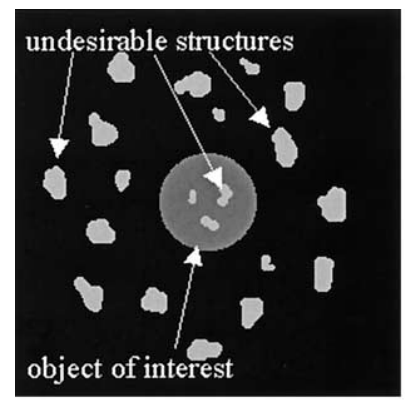

(a)

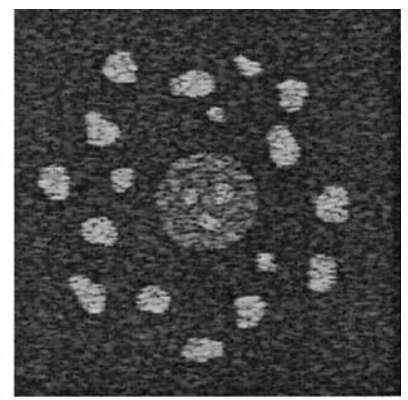

(b)
Fig. 5. (a) The raw synthetic image of size $256 \times 256$ used in this study (i.e., the synthetic image before corruption by the simulated speckle. The object of interest is a circular disk of radius 32 centered at $(128,128)$ and the sporadic spots are added to act as the obstacles interfering with the snake deformation; (b) a synthetic image with simulated speckle.

image is pre-defined, the synthetic image serves for the exact performance evaluation of the proposed dual-snake model. To simulate the speckle and the undesirable structures in a clinical US image, the synthetic image is composed of the object of interest, the sporadic spots and the simulated speckle. Figure $5 \mathrm{a}$ demonstrates the raw synthetic image of size $256 \times 256$ used in this study (i.e., the synthetic image before corrupted by the simulated speckle. The object of interest is a circular disc of radius 32 centered at $(128,128)$. The sporadic spots are added to act as the obstacles interfering with the snake deformation.

To simulate the speckle in a displayed US image, the speckle is simulated by generating the echo envelope of the fully developed speckle, followed by the nonlinear log-compression. The fully developed speckle is generated following the speckle simulation approach used by $\mathrm{Li}$ and O'Donnell (1994). More specifically, the speckle is modeled as a random walk in the complex plan. Each step in the random walk stands for the signal received by the transducer from a scatter in the resolution volume. With a high effective scatter density, the real and the imaginary parts of the summed signal may be individually modeled by a Gaussian distribution, which leads to a Rayleigh-distributed amplitude of the summed signal. It is assumed that the scanner is a 128-element linear array with the interelement space of $0.25 \mathrm{~mm}$. The central frequency and the bandwidth are $3 \mathrm{MHz}$ and $1 \mathrm{MHz}$, respectively. The axial direction is along the vertical axis of the simulated image. The shape of the envelope of the axial response is assumed to be Gaussian. The lateral response is derived by Fourier-transforming the aperture function with the assumption of a continuous-wave model. The point spread function of the scanner is assumed to be spatially invariant. 


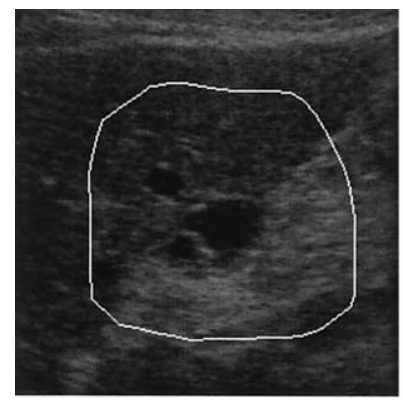

(a)

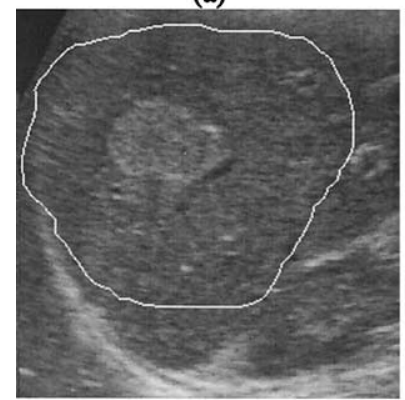

(c)

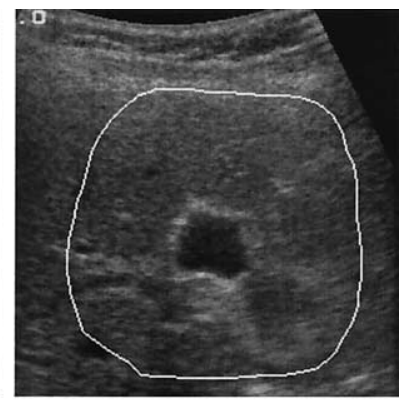

(b)

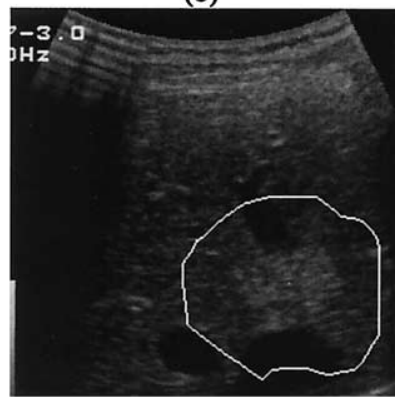

(d)
Fig. 6. Image clips of four testing images containing the tumors, in which the white contours are the initial outer snakes used by the proposed dual snake model for these four images.

Figure 5b demonstrates a synthetic image with simulated speckle. The contrast-to-noise ratio $(C N R)$ of Fig. $5 b$ is about 2.2. The CNR is defined as:

$$
C N R=\frac{\left|g_{f}-g_{b}\right|}{\max \left(\sigma_{\mathrm{f}}, \sigma_{\mathrm{b}}\right)},
$$

where $\sigma_{f}$ and $\sigma_{b}$ are the SDs of the speckle within and outside the disk, excluding the undesirables, and $g_{f}$ and $g_{b}$ are the grey levels of the disk and the background. To generate the synthetic images with various $C N R$ s for performance evaluation, $g_{f}$ and $g_{b}$ will be adjusted in the design of the synthetic images. For simplicity, the grey level of the undesirables, $g_{n}$, is set to $g_{n}=g_{f}+20$ so that the edge strength of the undesirables would be larger than that of the object of interest.

Although the synthetic image offers a known boundary for exact performance evaluation, the clinical US image provides the realistic complex image condition for evaluating the capability of the proposed dual-snake model. The desired boundary for the object-of-interest in a clinical US image is manually drawn by the experienced medical doctors. Because manual boundary delineation is quite a subjective process, the delineated boundary may be different for different medical doctors, or even for the same medical doctor at different times. Therefore, even though it is valuable to carry out perfor-

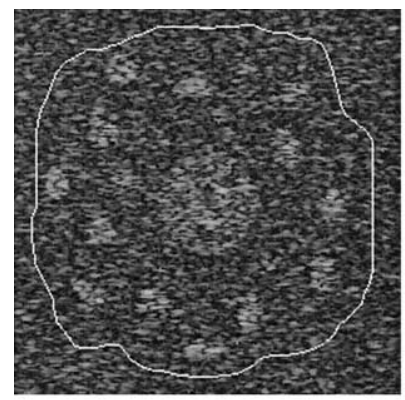

(a)

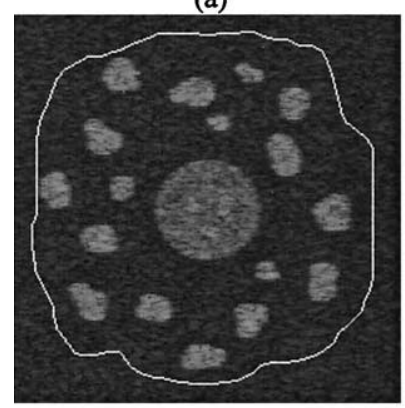

(c)

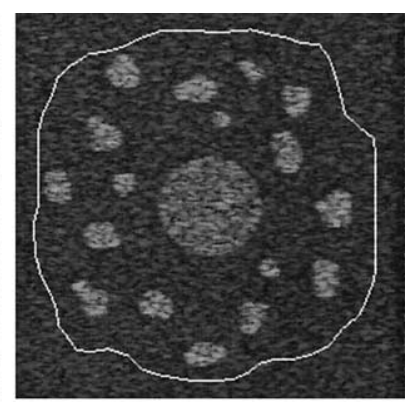

(b)

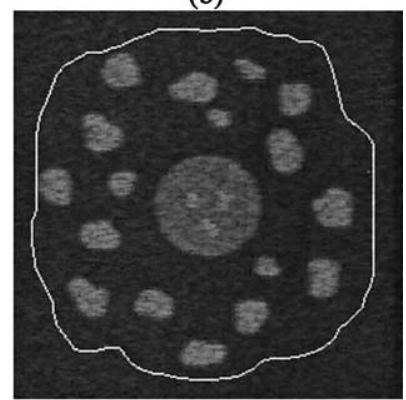

(d)
Fig. 7. Four of the ten synthetic images used in this paper with (a) $C N R=0.5$; (b) $C N R=2$; (c) $C N R=4$; and (d) $C N R=6$, respectively. The white contours are the initial outer snakes.

mance evaluation on the clinical US images, it is more appropriate to regard the analysis results as a reference.

To examine the ability of the proposed snake model in penetrating the false edges of the undesirables, we have carefully selected 19 US images so that each US image contains a hepatic tumor surrounded by some undesirables. These images were captured from the RGB outputs of a Toshiba SSA-380A clinical US imaging system through the frame grabber card, Meteor-II card, made by the Matrox Electronic System Ltd., Dorval, Québec, Canada. The operating frequency was $3 \mathrm{MHz}$. All images were stored in the BMP format with 8-bit resolution for each color channel. Among these 19 tumors, 11 of them were hypoechoic and the others were hyperechoic. For example, 2 hypoechoic and 2 hyperechoic tumors are shown in Fig. 6a-b and in Fig. 6c-d, respectively. One may see that each tumor has some undesirables in its vicinity.

\section{IMPLEMENTATION RESULTS AND DISCUSSIONS}

To evaluate the performance of the proposed dualsnake model, 10 synthetic images with $C N R=\{0.5,1,2$, $3,4,5,6,7,8,9\}$ were employed. All initial contours in this study have been placed quite far away from the desired boundaries, to test the capability of deforming from a distant initial contour. To ensure the same initial 
condition, all synthetic images will employ the same initial contour. As examples, Fig. 7a-d show four synthetic images with $C N R=\{0.5,2,4,6\}$, respectively. The white contours in Fig. $7 \mathrm{a}-\mathrm{d}$ are the initial contours, which will be used as the initial outer snakes. Recall that the initial inner snake for the proposed dual-snake model is a single point located at the center of mass of the ROI. One may observe that both of the inner and the outer snakes need to overcome the interference, not only from the speckle but also from the undesirables.

Taking the synthetic image with $C N R=2$ as an example, Fig. 8a illustrates the moving paths of the snaxels. The snaxels were selected for every 13 snake elements in this case. Figure $8 b$ shows the image despeckled by the MTM filter; $8 \mathrm{c}$ is the edge strength map obtained by applying the Sobel operator to $8 \mathrm{~b}$, from which one may observe that the edge strengths of the undesirables are stronger than that of the desired boundary. Figure $8 d$ gives the edge map containing the candidate edge points determined from the edge strength map; $8 \mathrm{e}$ demonstrates connected components determined from the dilated edge map. The grey level of each connected component is proportional to the size of the connected component. Figure $8 \mathrm{f}$ shows the weighted edge-strength map, which has been masked by the dilated edge map and weighted by the sizes of the connected components. It is clear that the edge strength of the desired boundary has become stronger than that of the false edges after being weighted by the size of the connected components. Through independent deformation, the deformation results at the end of the first stage for the outer and the inner snakes are given in Fig. 8g, which are illustrated as the outer (white) and the inner (grey) curves, respectively. Figure $8 \mathrm{~h}$ shows the deformation result derived at the end of the second stage by incorporating the stability measure and the $D S$-potential energy. At this moment, both snakes are supposed to have met each other completely. The final result achieved at the end of the third stage is given in Fig. 9b.

For visual inspection, Fig. 9a-d presents the final derived boundary through three-stage deformation for the four synthetic images given in Fig. 7a-d, respectively. Clearly, they have not only penetrated the hindrance from the speckle and the undesirables, but also have attained reasonable boundaries for the object of interest. Quantitatively, the mean distances between the derived boundaries and the desired boundaries for Fig. $9 \mathrm{a}-\mathrm{d}$ are $1.89,0.86,0.81$ and 0.79 pixels, respectively. For all 10 tested synthetic images, the mean, the maximum and the SD of the distances from all snake elements of the derived boundary to the desired boundary are plotted in Fig. 10, labeled as "mean," "max" and "Std," respectively. Generally speaking, the performance of the proposed dual snake model improves as the CNR in-

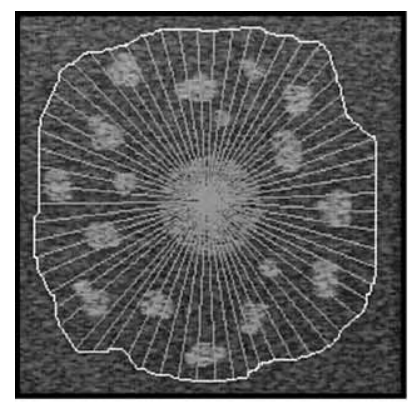

(a)

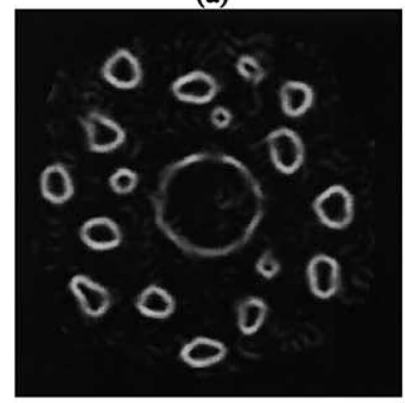

(c)

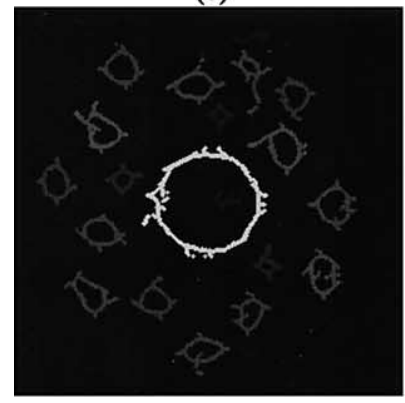

(e)

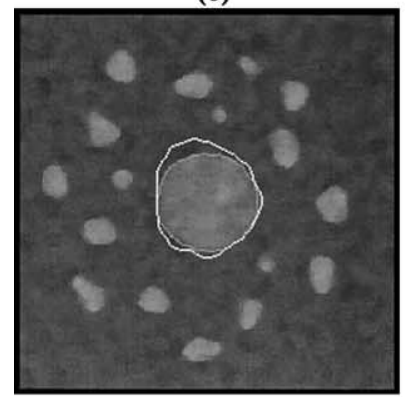

(g)

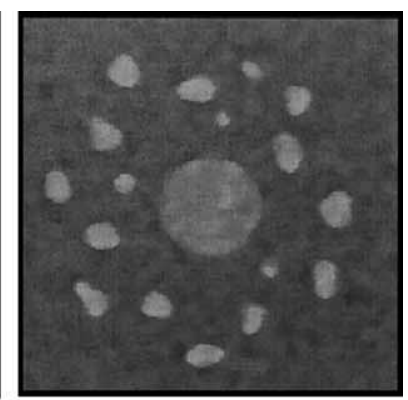

(b)

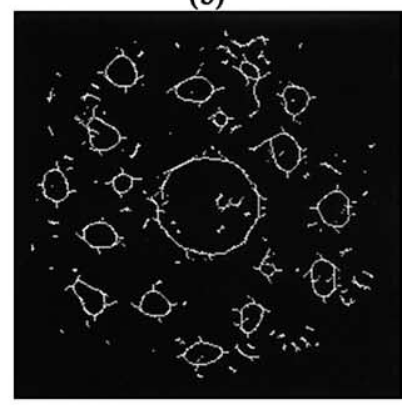

(d)

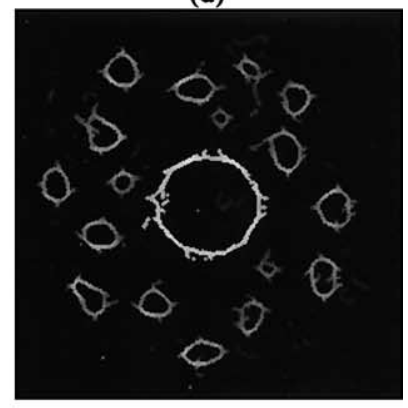

(f)

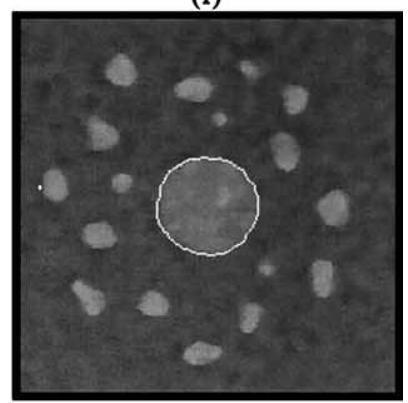

(h)
Fig. 8. (a) A synthetic image with $C N R=2$ and the snaxel moving paths; (b) the image despeckled by the MTMF filter; (c) the edge-strength map; (d) the edge map containing the candidate edge points; (e) the connected components; (f) the weighted edge-strength map; (g) the result of the first stage; and (h) the result of the second stage.

creases and is stabilized roughly after $C N R \geq 2$. For $C N R \geq 1$, the mean distance would be smaller than 1.4 pixels, and most snake elements are within 2.7 pixels of the desired boundary. Although, when $C N R=0.5$, the 


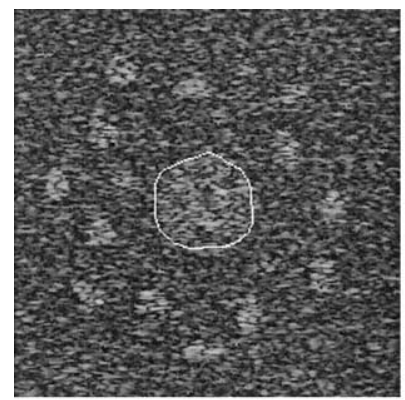

(a)

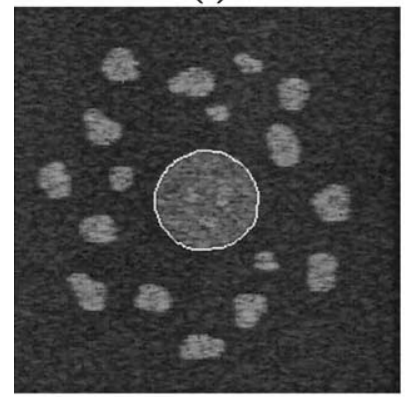

(c)

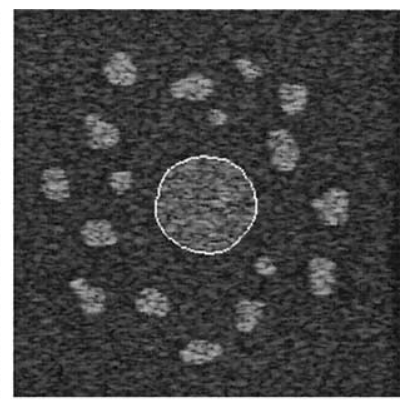

(b)

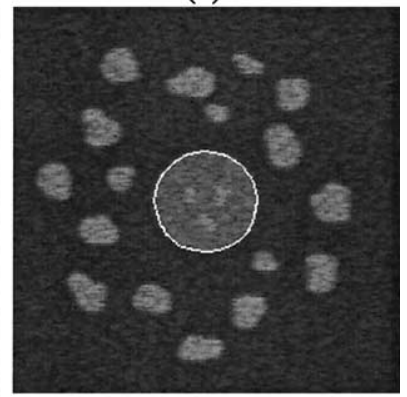

(d)
Fig. 9. The final derived boundary through three-stage deformation for the four synthetic images given in Fig. 7a-d, respectively. (a) $C N R=0.5$; (b) $C N R=2$; (c) $C N R=4$; and (d) $C N R=6$.

distance to the desired boundary may be as large as 5.1 pixels, it is a really difficult case because the object of interest is hardly visible.

For the synthetic images, the deviation of the derived boundary from the desired boundary may be imputed to two factors. One is the intrinsic blurring effect caused by the point-spread function of the simulated scanner, which would slightly enlarge the hyperechoic objects in the synthetic images. The other is the interference of the speckle, which may seriously degrade the

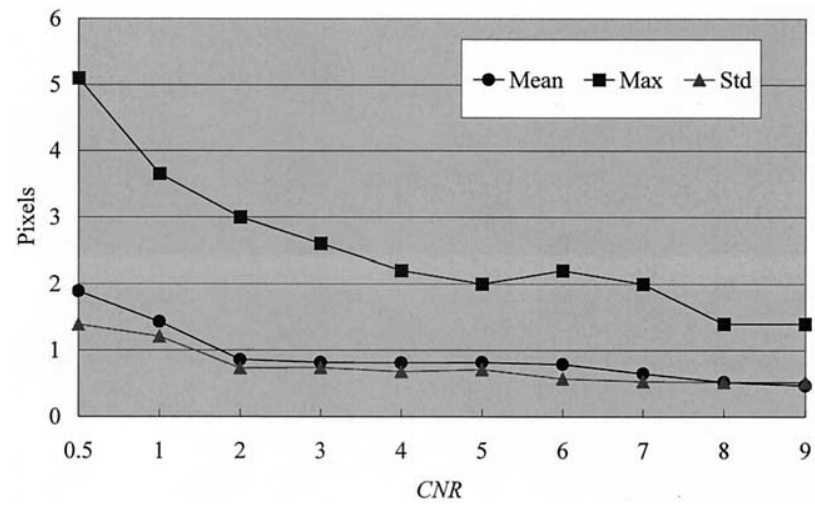

Fig. 10. The mean, the maximum and the SD of the distances from all snake elements of the derived boundary to the desired boundary for the 10 synthetic images, which are labeled as "mean," "max" and "Std," respectively.

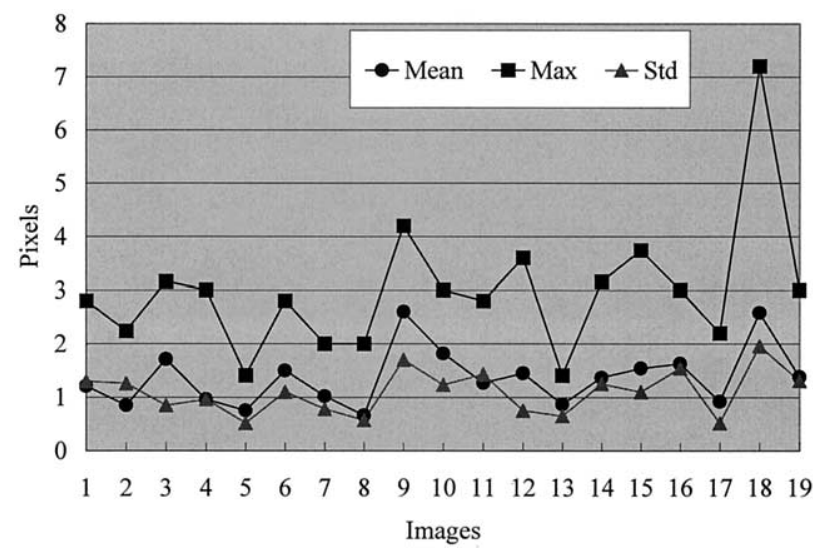

Fig. 11. The performances of the proposed dual-snake model for the 19 clinical US images, in which "mean," "max" and "Std" stand for the mean, the maximum and the SD of the distances from all snake elements of the derived boundary to the desired boundary.

edge definition of the desired boundary, especially when the $C N R$ is low. It was observed in our study, that the derived boundaries deviate from the desired boundary inward and outward randomly when $C N R=0.5$ and 1, mainly due to the interference of the noise. As the $C N R$ increases, because the interference of the speckle becomes more manageable by the MTM filter, the derived boundaries tend to deviate from the desired boundary outward, due to the blurring effect.

To investigate the effect of the false edges on the proposed snake model, for all 19 clinical US images, the initial contour has been intentionally placed reasonably far away from the desired boundary. As examples, the initial contours for the four typical hepatic tumors shown in Fig. 6 are marked as the white contours, each of which encloses some undesirables. One may observe that the four ROIs contain either large undesirables with strong edges (Figs. 6a, b and d) or many sporadic spots with weak edges (Fig. 6c). Moreover, some tumor boundaries may be too weak to be well defined (e.g., Fig. 6d). As a result, the performances of the proposed dual snake model for the 19 clinical US images are plotted in Fig. 11. Similarly, "mean," "max" and "std" stand for the mean, the maximum and the SD of the distances from all snake elements of the derived boundary to the desired boundary. Statistically, the mean and the SD of "mean", "max" and "std" are summarized in Table 1. In general, the proposed snake model has successfully penetrated the interference of the false edges and achieved the mean and the maximum distances less than 1.9 and 4.2 pixels, respectively, for most testing images. Furthermore, most snake elements in the derived boundaries are within 3 pixels of the desired boundaries (i.e., "mean" + "std").

For visual inspection, Fig. 12a-d give the boundaries 
Table 1. The mean and the SD of the three curves (i.e., mean, max and std, illustrated in Fig. 11

\begin{tabular}{lccc}
\hline & Mean & Max & Std \\
\hline Mean & 1.37 & 2.98 & 1.09 \\
SD & 0.55 & 1.26 & 0.41 \\
\hline
\end{tabular}

Unit $=$ pixels.

of the tumors derived by the proposed dual snake model for Fig. 6a-d, respectively. In contrast, Fig. 13a-d demonstrates the boundaries drawn by a medical doctor for Fig. $6 \mathrm{a}-\mathrm{d}$, respectively. The "mean" values for these four images (i.e., Fig. 6a-d) are 0.96, 0.66, 1.45 and 2.58 pixels. One can see that, even though there are strong false edges in the vicinity of the tumors, the distant initial contours have still overcome their interference and come close enough to the desired boundaries. From both the visual perception and the mean distances between the derived and the desired boundaries, it may be reasonable to say that the derived boundaries closely follow the desired boundaries.

The discrepancies between the derived and the desired boundaries may be ascribed to at least three factors. The first one is the false edges due to the speckle, the tissuerelated textures and the artefacts. This factor is particularly crucial when the $C N R$ is low. For example, the "max" value for image 18 is as large as 7.2 pixels because the $C N R \mathrm{~s}$ around the upper-left and the lower-right corners of the

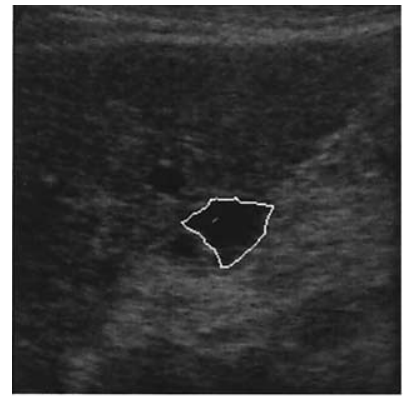

(a)

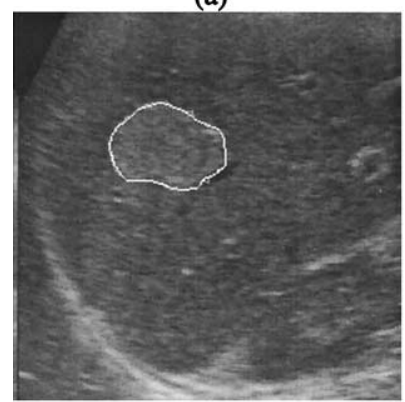

(c)

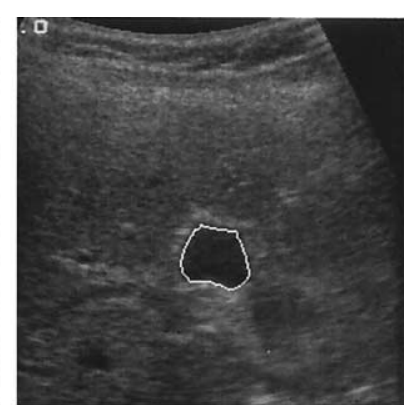

(b)

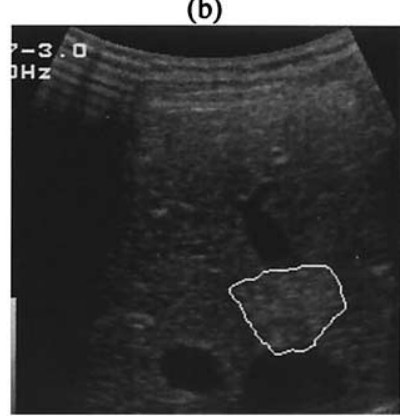

(d)
Fig. 12. The boundaries of the tumors derived by the proposed dual-snake model for Fig. 6a-d, respectively.

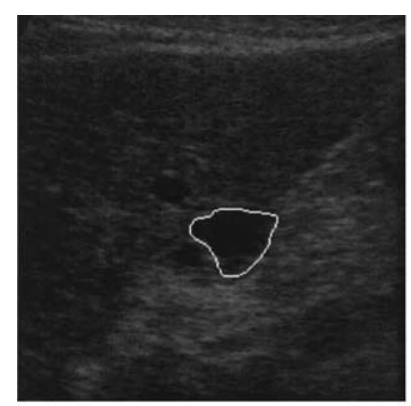

(a)

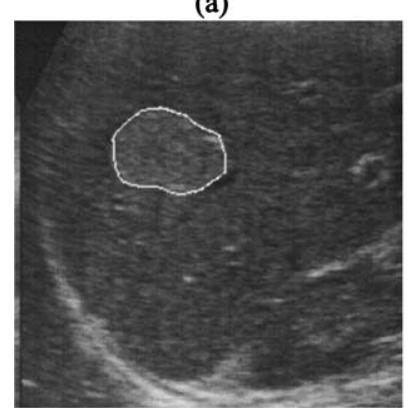

(c)

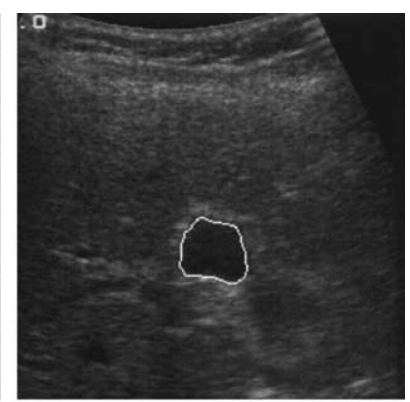

(b)

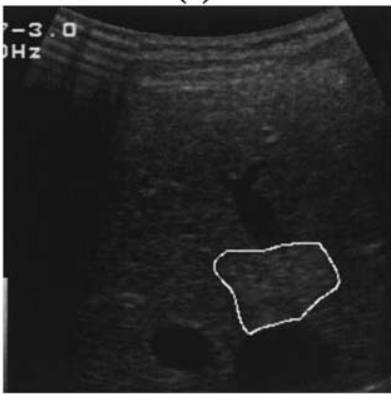

(d)
Fig. 13. The boundaries drawn by a medical doctor for Fig. 6a-d, respectively.

tumor in Fig. $6 \mathrm{~d}$ are only about 0.8 . The second factor is that the edges defined by visual perception may be different from those defined by mathematical models such as gradients, zero-crossings, and so on. The third factor is that the visual definition may vary with medical doctors, which may be partly due to the ill-defined edges and partly to doctors' subjective opinions of the definition of a tumor boundary. An ill-defined boundary can lead to quite different decisions of medical doctors on a tumor boundary, as demonstrated in Chen et al. (2000).

Because of the randomness inherent in these three factors, the boundaries derived for the clinical US images generally appear to deviate from the manually drawn boundaries inward and outward randomly. In theory, the blurring effect caused by the point spread function of the clinical scanner may also degrade the performance of the proposed dual-snake model. However, this degradation effect is not evident in the experimental results, because the true boundaries are not known and the manually delineated boundaries can only be used as a reference. Therefore, the area-expanding phenomenon due to the blurring effect shown in the derived boundaries of the synthetic images with $C N R \geq 2$ is not obvious for the clinical US images.

\section{CONCLUSIONS}

With the emphasis on allowing a distant initial contour, which is a valuable property for practical applications, 
in this paper, we have proposed a new deformable model for boundary extraction from the US images. By integrating the ideas of the far-reaching external force, the dual-snake model, and the discrete concept, the proposed dual-snake model has achieved a high penetrability through the interference of various undesirables, such as the speckle, the tissue-related textures and the artefacts. A new external force, called discrete gradient flow (DGF), has been devised to provide the proposed dual-snake model with an effective far-reaching attraction force toward the desired boundary. To enhance the image force of the object-of-interest, a new weighting scheme based on the connected component has been developed to enforce the conventional edge strength for the object of interest. To emphasize the role of the edge points, a new stability index, defined as a function of the distances from the snaxels to the nearest edge points, has been proposed to evaluate the stability of two underlying snakes.

From the experimental results, it has been shown that the proposed dual-snake model not only has the ability to deform from a distant initial contour, but also has attained reasonable boundaries for both the simulated and the clinical US images. For the synthetic images, when $C N R \geq 1$, the mean distance from the derived boundary to the desired boundary would be smaller than 1.4 pixels, and most snake elements are within 2.7 pixels of the desired boundary. For the clinical US images, the proposed snake model has achieved the mean and the maximum distances of less than 1.9 and 4.2 pixels, respectively, for most testing images. Furthermore, most snake elements in the derived boundaries are within 3 pixels of the desired boundaries.

\section{REFERENCES}

Boukerroui D, Basset O, Guerin N, Baskurt A. Multiresolution texture based adaptive clustering algorithm for breast lesion segmentation. Eur J Ultrasound 1998;8:135-144.

Chalana V, Linker DT, Haynor DR, Kim Y. A multiple active contour model for cardiac boundary detection on echocardiographic sequences. IEEE Trans Med Imaging 1996;15:290-298.

Chen CM, Lu HHS. Sonographic tumor boundary detection for imageguided brain surgery. Proceedings of the Fifth World Multi-Conference on Systemics, Cybernetics and Informatics, Orlando, FL, USA, July 22-25, 2001, pp. 339-344 (in press).

Chen CM, Lu HHS, Lin YC. An early vision based snake model for ultrasound image segmentation. Ultrasound Med and Bio 2000; 26(2):273-285.

Cohen LD. On active contour models and balloons. Comput Vision Graphics Image Processing Image Underst 1991;53(2):211-218.

Cohen LD, Cohen I. Finite-element methods for active contour models and balloons for 2-D and 3-D images. IEEE Trans Pattern Anal Machine Intel 1993;15:1131-1147.

Fan L, Braden GA, Herrington DM. Nonlinear wavelet filter for intracoronary ultrasound images. Proceedings of the 1996 23rd Annual Meeting on Computers in Cardiology, 1996;41-44.

Giraldi GA, Goncalves LM, Oliveira AF. Dual topologically adaptable snakes. Proceedings of the 5th Joint Conference on Inform Science, 2000;2:103-106.
Gunn SR, Nixon MS. A robust snake implementation; A dual active contour. IEEE Trans PAMI 1997;19:63-68.

Haas C, Ermert H, Holt S, et al. Segmentation of 3-D intravascular ultrasonic images based on a random field model. Ultrasound Med Biol 2000;26:297-306.

Hamdan HM, Youssef AB, Rasmy ME. The potential of mathematical morphology for contour extraction from ultrasound images. Proceedings of the 18th Annual International Conference of the IEEE EMBS 1996;881-882.

Heckman T. Searching for contours. Proc SPIE 1996;2666:223-232.

Iwase M. 3D Echocardiography and semi-automatic border detection to assess left ventricle volume and function: Comparison with MRI. Circulation 1994;90:1337.

Kass M, Witkin A, Terzoulos D. Snake: Actour contour models. Int J Comput Vision 1987;1:321-331.

Kerschner M. Homologous twin snakes integrated in a bundle block adjustment. Proceedings of Symposium on Object Recognition and Scene Classification from Multispectral and Multisensor Pixels 1998 (http://www.ipf.tuwien.ac.at/veroeffentlichungen/mk_p_isprs98/paper3.htm).

Kotropoulos C. Nonlinear ultrasonic image processing based on signak-adaptive filters and self-organizing neural networks. IEEE Trans Image Processing 1994;3:65-77.

Lee YH, Kassman SA. Generalized median filtering and related nonlinear filtering techniques. IEEE Trans Acoust Speech Signal Processing 1985;33:672-683.

Lefebvre F, Berger G, Laugier P. Automatic detection of the boundary of the calcaneous from ultrasound parametric images using an active contour model; Clinical assessment. IEEE Trans Med Imaging 1998; 17:45-52.

Li PC, O'Donnell M. Improved detectability with blocked element compensation. Ultrason Imaging 1994;16:1-18.

Mikic I, Krucinski S, Thomas JD. Segmentation and tracking in echocardiographic sequences: Active contours guided by optical flow estimates. IEEE Trans Med Imaging 1998;17:274-284.

Niessen WJ, ter Haar Romeny BM, Viergever MA. Geodesic deformable models for medical image analysis. IEEE Trans Med Imaging 1998;17:634-641.

Pathak SD, Chalana V, Kim Y. Interactive automatic fetal head measurements from ultrasound images using multimedia computer technology. Ultrasound Med Biol 1997;23:665-673.

Sarty GE, Liang W, Sonka M, Pierson RA. Semiautomated segmentation of ovarian follicular ultrasound images using a knowledgebased algorithm. Ultrasound Med Biol 1998;24:27-42.

Shekhar R, Cothren RM, Vince DG, et al. Three-dimensional segmentation of luminal and adventitial borders in serial intravascular ultrasound images. Comput Med Imaging Graphics 1999;23:299-309.

Skaane P, Engedal K. Analysis of sonographic features in the differentiation of fibroadenoma and invasive ductal carcinoma. AJR 1998;170:109-114.

Solaiman B, Roux C, Rangayyan RM, Pipelier F, Hillion A. Fuzzy edge evaluation in ultrasound endosonographic images. Proceedings of the 1996 Canadian Conference on Electrical and Computer Engineering, 1996;335-338.

Terzoulos D, Witkin A, Kass M. Constraints on deformable models: Recovering 3-D shape and nonrigid motion. Artif Intelligence 1988;36:91-123.

Thomas JG, Peters RA, Jeanty P. Automatic segmentation of ultrasound images using morphological operators. IEEE Trans Med Imaging 1991;10:180-186.

Xu C, Prince JL. Snakes, shapes, and gradient vector flow. IEEE Trans Image Processing 1998;7:359-369.

Yoshio A. Prostatic cancer staging by ultrasound and digital examination. In: Resnick M, Hiroki W, Karr J, eds. Diagnostic Ultrasound of the Prostate. Proc. 1st International Workshop on Diagnostic Ultrasound of the Prostate. New York: Elsevier, 1998:48-52.

Zimmer Y, Tepper R, Akselrod S. A two-dimensional extension of minimum cross entropy thresholding for the segmentation of ultrasound images. Ultrasound Med Biol 1996;22:1183-1190. 